\title{
3D RECONSTRUCTION OF IRREGULAR BUILDINGS AND BUDDHA STATUES
}

\author{
ZHANG Kai *, LI Ming-ju
}

\author{
Provincial Geomatics Centre of Jiangsu, 210013 Nanjing, China - thesummernight@gmail.com \\ limingju@sina.com
}

\section{Commission}

\begin{abstract}
KEY WORDS: Cultural Heritage, Laser Scanning, 3D Reconstruction, Irregular Ancient Buildings, Buddha Statues, Data
\end{abstract} Acquisition, Modelling and Texture Mapping

\begin{abstract}
:
Three-dimensional laser scanning could acquire object's surface data quickly and accurately. However, the post-processing of point cloud is not perfect and could be improved. Based on the study of 3D laser scanning technology, this paper describes the details of solutions to modelling irregular ancient buildings and Buddha statues in Jinshan Temple, which aiming at data acquisition, modelling and texture mapping, etc. In order to modelling irregular ancient buildings effectively, the structure of each building is extracted manually by point cloud and the textures are mapped by the software of 3ds Max. The methods clearly combine 3D laser scanning technology with traditional modelling methods, and greatly improves the efficiency and accuracy of the ancient buildings restored. On the other hand, the main idea of modelling statues is regarded as modelling objects in reverse engineering. The digital model of statues obtained is not just vivid, but also accurate in the field of surveying and mapping. On this basis, a 3D scene of Jinshan Temple is reconstructed, which proves the validity of the solutions.
\end{abstract}

\section{INTRODUCTION}

Jinshan Temple is located in the northwest of national historical and cultural city of Zhenjiang. It is famous for legends and fairy tales such as "water overflows Jinshan", and has always been a symbol of Zhenjiang. In more than 1600 years of history changes, Jinshan Temple was rebuilt many times, which embodies the art and the main features of architecture in Tang, Song, Yuan, and Qing Dynasty. For accurately and truly representing characteristics of Jinshan temple's cultural landscape, and meeting the requirements of the cultural heritage conservation of, planning and construction, a three-dimensional digital reconstruction of the Jinshan Temple has been carried out.

There are two typical objects in Jinshan Temple. One is irregular buildings which roofs stretch out, and another one is Buddha statues which have a smooth and curve surface. The traditional technology of 3D modelling is very difficult to model their structure accurately and map their texture vividly. In recent years, the development of 3D laser scanning technology has shown its unique advantages in such aspects as complex three-dimensional information acquisition. Due to the complexity of the point cloud, the data processing has become a bottleneck of its wide application. Thus in the field of cultural heritage conservation and virtual reality, the product of 3D laser scanning stays in the form of point cloud for digital preservation results or just single object model. 3D scenes of large-scale are reconstructed rarely.

In this paper, based on the study of 3D laser scanning technology, the solutions to modelling irregular ancient buildings and statues are presented; and the digital 3D scene of Jinshan Temple is built, which could promote the application of 3D laser scanning technology.

\section{3D LASER SCANNING TECHNOLOGY}

LiDAR is short for "Light Detection and Ranging", also called 3D laser scanning technology, which directly uses acquired data, such as location, distance, angle, etc. to obtain coordinate of object's surface data, realizing extraction of surfacing information and 3D scene construction. According to different carriers, LiDAR can be divided into airborne, vehicle, static terrestrial and other types, in which static terrestrial laser scanning is well used of acquiring accurate data of objects.

The design of 3D laser scanning system varies with different applications. It mainly includes laser ranging module and laser scanning module, and integrates CCD, internal instrument control and calibration systems. The principle of laser ranging has three types: pulse-ranging method, phase-ranging method, and triangulation method. Pulse-ranging method is expert in long-distance data acquisition, and is widely used in the field of surveying and mapping, which by way of emitting laser; calculate distance according to the time between the laser transmitter and receiver. Phase-ranging method is fit for shortdistance data acquisition, which by means of principle of optical interference. Its measure range is generally less than $50 \mathrm{~m}$, and its speed of measure can reach 10000 to 50000 points per second. Triangulation method refers to using a stereo camera and structured light sources, establishes stereo projection, thus obtain the distance. Triangulation method is fit for shortdistance measurement, which range is less than $20 \mathrm{~m}$, and speed is 100 points per seconds.

The digital reconstruction of Jinshan Temple is divided into two components. The first component is the irregular ancient buildings and terrain. We used a pulsed Z420i laser scanning system, which had long distance and wide range. Secondly, we used Focus3D system to get data of the eighteen statues and

\footnotetext{
* Corresponding author. This is useful to know for communication with the appropriate person in cases with more than one author.
} 
other objects indoor. The Focus3D system that based on phaseranging method had fast scan speed and high density. The main parameters of the two instruments are as shown in Table 1.

\begin{tabular}{|l|l|l|}
\hline & Riegl LMS Z420i & Faro Focus3D \\
\hline Range & $2-1000 \mathrm{~m}$ & $0.6-120 \mathrm{~m}$ \\
Angle & Level $360^{\circ}$, vertical $80^{\circ}$ & Level $360^{\circ}$, vertical \\
& 12000 points / second & $305^{\circ}$ \\
Speed & maximum & 976000 points / \\
& second maximum \\
Accuracy & $\pm 5 \mathrm{~mm}$ (average) & $\pm 2 \mathrm{~mm}(10 \mathrm{~m}$ and \\
& Integrated CCD( registered, & Built in 7000 pixel \\
Camera & replaceable) & camera \\
Weight & $14.5 \mathrm{~kg}$ & $5.0 \mathrm{~kg}$ \\
\hline
\end{tabular}

Table 1. Main parameters of the two instruments

\section{3D RECONSTRUCTION OF IRREGULAR BUILDINGS}

The existing acquisition of 3D laser scanning technology has been mature; the post-process can be improved however. Aimed to the complex irregular ancient buildings, combined with the traditional modelling methods, the following solution to modelling was adopted, which is: the buildings were scanned firstly; and then the buildings' structure was extracted manually; after textures were mapped in the software of 3ds Max, the 3D reconstruction of ancient buildings of Jinshan Temple was achieved.

\subsection{Data acquisition}

The acquisition of point cloud includes planning and scanning.

3.1.1 Planning: Under the limitation of environment and the shape of objects, the overall point cloud of complex objects cannot be obtained in one-time. Therefore, proper planning should be carried out for a multi-station, multi-angle data scans. The basic principle is to obtain the surface features as little stations as possible, and consider the overlap between stations and reduce the impact of environment.

The plan of stations should be performed hierarchically considering specific terrain conditions. For example, the Mahavira Palace in Jinshan Temple was made up to seven stations from various locations and angles, while fewer stations were set for the side buildings. After investigation and comprehensive planning, more than 100 stations from the outside to inside were arranged in the data acquisition of Jinshan Temple.

3.1.2 Scanning: Firstly, the panorama of scene around the station should be scanned with low-accuracy. Secondly, a certain interested area could be selected, and scanned finely. The scanning density should be determined according to the architectural structure, size, and distance from the instrument. The remains scans should be performed by the planning, until the whole data collection is complete.

\subsection{Data processing}

Due to measurement error, view occlusion and other reasons, point cloud acquired by 3D laser scanning has a lot of defects, such as noise, leak, non-matched data, etc. The raw data should be processed to eliminate these defects. Data processing generally includes data registration, data partition, and data simplification and so on.

Each scan in different station is in a separate coordinate system. All scans should be registered to a unified coordinate system in order to get a complete scene. In this project, scans of Jinshan Temple were specifically registered in RiSCAN Pro software, using feature points extracted manually as correspondence points. This method brings out different precision and the precision has better mainly below $2 \mathrm{~cm}$. If it exceeds, the feature points should be selected again.

When dealing with point cloud in 3ds Max, it will consume a significant amount of resources. The better way is to carry out spatial partition and data simplification. Spatial partition means each individual building of the point cloud should be divided into parts; and then separately imported into $3 \mathrm{ds}$ max. If the data of single building is still large, data simplification like resample or reducing manually is required.

\subsection{Modelling and texture mapping}

The basic idea of 3D modelling is to generate fitting models automatically according to points. For example, points of a cylindrical geometry object could be fit to cylinder model. This approach can accurately restore the object's form; however it is confined to noise of point cloud and the algorithm of complex object in engineering. On the other hand, traditional 3D modelling method can simulate architecture without accuracy. On this basis, this paper proposes the technology which traditional modelling combined with the technology of point cloud is used to reconstruct the ancient buildings.

The specific method is: the location, form and size of buildings are determined by the cloud point, thus the main structure is built. This method bases on constructing geometric model manually, while the complicated post-processing of point cloud is simplified and the accuracy could be controlled. Finally, textures are mapped in the models. Figure 1 is a landscape of 3D Jinshan Temple reconstructed throughout this technology course.

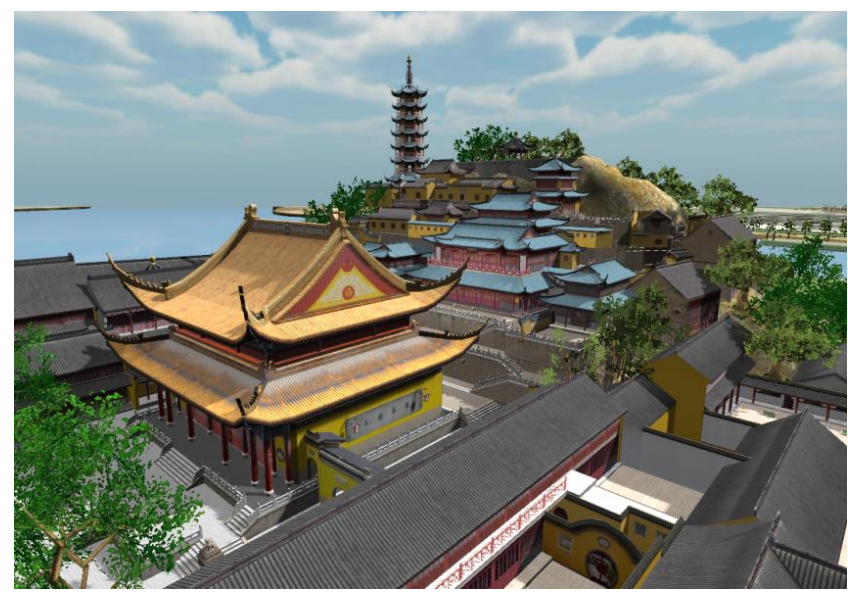

Figure1. A landscape of 3D Jinshan Temple

This method avoids the most complicated process of point cloud; common modellers are capable for this job. It clearly combines 3D laser scanning technology with traditional modelling methods, and greatly improves the efficiency and accuracy of the irregular buildings restored. 


\section{3D RECONSTRUCTION OF BUDDHA STATUES}

In all viharas, the Mahavira Palace occupies an important position. The previous Mahavira Palace in Jinshan Temple was built in Ming Dynasty, which had destroyed in 1948. The design of new Mahavira Palace has a style of grandness and elegance. Buddhas of Three Periods and the Eighteen Arhats are exquisite, which can be considered as artistic gems. The indoor of the Mahavira Palace takes an important part in the digital preservation of Jinshan Temple.

The digital model of statues as the Eighteen Arhats can be constructed vividly by software as Maya, 3ds Max, etc. However, the reproduction just stays in the level of visualization, which cannot represent its real size in 3D dimensions. Base on the 3D laser scanning technology, this project studies a solution to modelling statues, which is different to the solution to modelling irregular ancient buildings from data acquisition, processing to modelling and texture mapping. The comparison is as shown in Table 2.

\begin{tabular}{|c|c|c|}
\hline & $\begin{array}{l}\text { Irregular Ancient } \\
\text { buildings }\end{array}$ & Buddha Statues \\
\hline Scanner & Long-range & Short-range \\
\hline Reflector & Unused & Spherical reflector \\
\hline $\begin{array}{l}\text { Image } \\
\text { collection }\end{array}$ & $\begin{array}{l}\text { Captured from digital } \\
\text { camera }\end{array}$ & $\begin{array}{l}\text { Captured from digital } \\
\text { camera }\end{array}$ \\
\hline $\begin{array}{l}\text { Data } \\
\text { registration }\end{array}$ & $\begin{array}{l}\text { Correspondence } \\
\text { points from feature } \\
\text { points selected } \\
\text { manually }\end{array}$ & $\begin{array}{l}\text { Correspondence points } \\
\text { from the centre of the } \\
\text { reflector extracted } \\
\text { automatically }\end{array}$ \\
\hline $\begin{array}{l}\text { Precision of } \\
\text { registration }\end{array}$ & $2 \mathrm{~cm}$ & $3 \mathrm{~mm}$ \\
\hline Modelling & $\begin{array}{l}\text { Extract structure } \\
\text { based on point cloud }\end{array}$ & Construct TIN \\
\hline Texture & $\begin{array}{l}\text { Mapping for each } \\
\text { polygon }\end{array}$ & $\begin{array}{l}\text { Mapping overall and } \\
\text { rendering }\end{array}$ \\
\hline
\end{tabular}

Table 2. Comparison of two solutions of 3D reconstruction to irregular ancient buildings and statues

\subsection{Data acquisition}

The Mahavira Palace is 20 meters high, 24.6 meters length and width, which internal space is compact. In order to obtain more comprehensive information of statues, the project used Faro Focus3D scanner, and set up a total of 12 stations and 18 spherical reflectors, which scan time for each station is about 9 minutes.

The function of spherical reflectors is similar to the control points in geodetic surveying, in which the layout directly determines precision of data registration. In the laid process, the need should meet the following requirement.

(1) All correspondence reflectors in adjacent stations do not lie on the same direction;

(2) The number of reflectors in adjacent stations should not less than 3 ; the optimum number is $4-5$.

At the same time, images of Buddha statues could be collected with digital camera.

\subsection{Data processing}

The centre of spherical reflectors can be extracted automatically via software of Scene, which can be used as correspondence points for data register. This method not just improves the efficiency of data register, but also improves the precision of data register.

The practice validated the average error of register of entire the Mahavira Palace was $3 \mathrm{~mm}$, which could fill the requirement of modelling. After all point cloud of the Mahavira Palace was obtained, each statue of Eighteen Arhats can be exported respectively.

\subsection{Modelling and texture mapping}

To generate geometrical model directly from point cloud is another key technical issues of this project. The principal way is to construct TIN from points; which means constructing automatically from point cloud to wire frame model. However, due to existing technology is immature; software blinded with the scanner could not solve the problem perfectly. Here, TIN was constructed by the software of Geomagic which mostly used in the field of reverse engineering. Practice has improved that the way can achieve good geometrical models in the condition of erasing noise, simplification, fill holes and repair operations.

Statues belonging to the ancient cultural relics have a wealth of geometric detail, and valuable archaeological information like colour, texture and text on the surface. Only the information was merged together, the digital model of the cultural relic is valuable. This project firstly takes overall mapping in Geomagic. This approach truly reflect the actual texture of the statues, however the defect is that it can be just mapped using single photo, which maybe come in stretch and missed in somewhere like the below of arms and the back of statues. For this reason, they were modified using Photoshop to obtain more complete textures. Finally, the models were rendered to add light effects in 3ds Max to achieve more realistic results.

As steps is shown in Figure 2 from point cloud to a model, the digital model of statues using the method is not just vivid in the field of animation, but also accurate in the field of surveying and mapping. The results can be used as digital preservation of cultural relics.

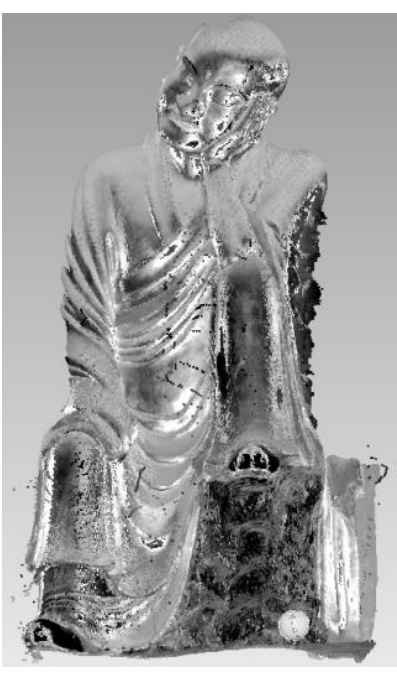

a - point cloud after treated

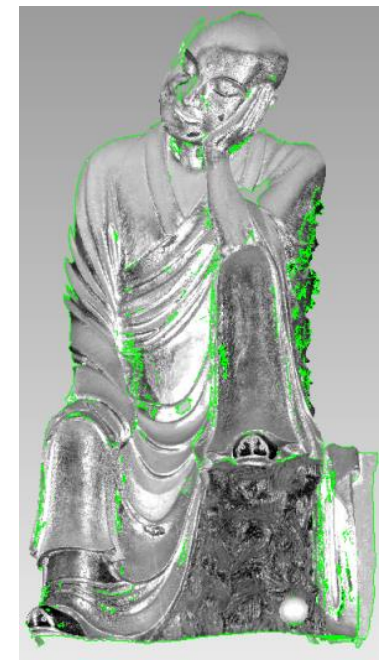

b - TIN 


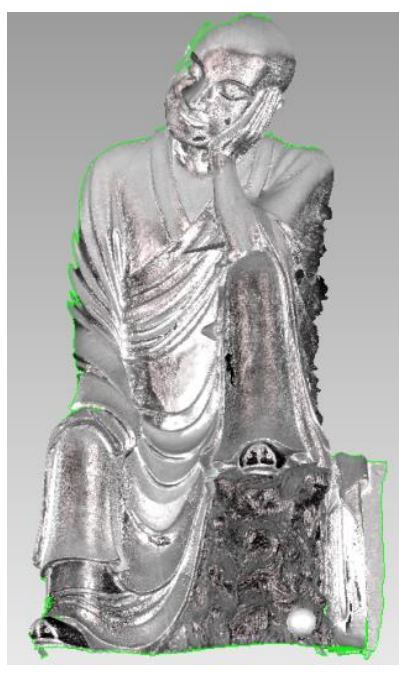

c - optimize TIN

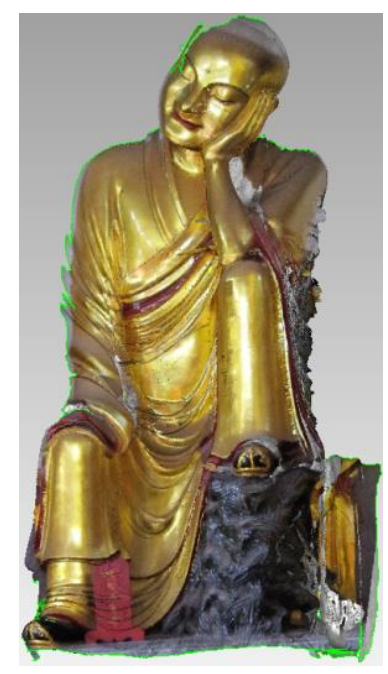

d - model textured

Figure 2. Steps of statues reconstruction

\section{CONCLUSION}

The laser scanning technology has been through a rapid development in many fields in recent years, and has become the primary means to acquire accurate data. However, the existing studies are mainly focused on the process of point cloud and modelling automatically, while the application on industry and cultural heritage conservation is need to be improved. Based on the research of the 3D laser scanning technology, this paper provides solutions individually aimed at modelling irregular buildings and statues, which simplify the complicated postprocessing of point cloud. Furthermore, a 3D scene of Jinshan Temple is reconstructed by using the solutions, which truly restored its architectural structure and interior statues' style. The achievement has the characters of high simulation and high accuracy, which could be used in planning and management of temple, tourism, heritage preservation and restoration.

\section{REFERENCE}

Zheng De-hua. The theory and method of the data processing based on 3D laser scanning point cloud data. Tongji University. 2005

Cheng Si-yuan. Geomagic Studio - Reverse engineering technologies and applications. Beijing: Tsinghua university press, 2010

Hu Shao-xing, Zha Hong-bin, Zhang Ai-wu. Modeling method for large-scale cultural heritage sites and objects using real geometric data and real texture data. In: Journal of System simulation, 2006, 18(4), pp. 951 - 954, 963 